\title{
Measurements of the Atmospheric Neutrino Flux at Super-Kamiokande
}

\author{
Euan Richard* \\ Institute for Cosmic Ray Research, the University of Tokyo \\ E-mail: richardeicrr.u-tokyo.ac.jp

\section{Kimihiro Okumura} \\ Institute for Cosmic Ray Research, the University of Tokyo \\ E-mail: okumuraeicrr.u-tokyo.ac.jp

\section{Takaaki Kajita} \\ Institute for Cosmic Ray Research, the University of Tokyo \\ E-mail: kajita@icrr.u-tokyo.ac.jp
}

\section{The Super-Kamiokande Collaboration}

Three direct measurements of the atmospheric $v_{e}+\bar{v}_{e}$ and $v_{\mu}+\bar{v}_{\mu}$ neutrino fluxes were performed using the Super-Kamiokande water Cherenkov detector: the directionally-integrated energy spectra, the azimuthal spectra, and the modulation of the fluxes with time over the 11-year solar cycle.

In particular, the energy spectra in the sub-GeV to $\mathrm{TeV}$ range was measured and compared to the predictions of various published flux models. While none of the models were strongly inconsistent with the data, some preference was seen for the HKKM11 model as the most realistic model.

The azimuthal analysis measured the east-to-west asymmetry in the neutrino flux, caused by the geomagnetic field, for both flavours at $>5 \sigma$. Measurements were made of the strength of the effect as functions of energy and zenith angle. There was also an indication that the alignment of the asymmetry was dependent on zenith angle, seen at the $2.2 \sigma$ level.

A search for a long-term correlation between the atmospheric neutrino flux and the solar magnetic activity cycle was performed, however the expected effect based on the HKKM model was calculated to be relatively small. An indication of a correlation was seen at the $1.1 \sigma$ level. During particularly strong solar activity events, known as Forbrush decreases, no theoretical prediction is available, but a deviation from the expected neutrino event rate is seen at the $2.4 \sigma$ level.

The 34th International Cosmic Ray Conference

30 July- 6 August, 2015

The Hague, The Netherlands

\footnotetext{
* Speaker.
} 


\section{Introduction}

Atmospheric neutrinos are generated from the decay of mesons produced in cosmic-ray interactions in the Earth's atmosphere, and are one of the main experimentally available neutrino sources. Since the first detection of the atmospheric neutrino in underground experiments in the 1960s [1, 2], further measurements of them brought the discovery of neutrino oscillation (and thus finite neutrino masses) in 1998 [3]. Since then the understanding of the oscillation parameters has improved quickly, by using the atmospheric [4] data in combination with solar [5, 6], reactor $[7,8,9,10]$, and accelerator $[11,12,13,14]$ sourced neutrinos.

The atmospheric neutrino sample spans a wide energy region, peaking at $O(\mathrm{MeV})$ but so far measured up to $O(\mathrm{PeV})$, and atmospheric neutrinos can be detected up to $\sim 13,000 \mathrm{~km}$ away from their initial creation point (i.e. after crossing the diameter of the Earth). They are thus a useful source as a varied and high-statistics neutrino beam, however to understand and use them as a sample, an accurate prediction of the expected flux depending on neutrino flavour, energy, direction, and time is required. For example, the discovery of neutrino oscillation was by comparison of their expected zenith angle distribution with and without oscillation applied. Furthermore, the atmospheric neutrino is not just a signal but also a background source to many other experiments, such as astrophysical neutrino, proton decay, and dark matter searches. An accurate prediction of the flux is thus of paramount importance to many current and future experiments. As one example, the planned Hyper-Kamiokande [15] experiment could use the atmospheric sample to uncover the neutrino mass hierarchy.

Current atmospheric neutrino flux predictions are given by Monte Carlo simulations by several groups, such as the HKKM [16], Fluka [17], and Bartol [18] models. These models are based on data such as the primary cosmic ray proton flux and the secondary muon flux, measured in the atmosphere by particle detectors on balloons and spacecraft, and hadron production measured in accelerator experiments. Direct experimental measurements of the flux energy spectra were made by the Frejus [19] collaboration (before neutrino oscillation was known), and more recently by the AMANDA-II $[20,21]$ and IceCube $[22,23,24]$ collaborations at higher energies (up to the $100 \mathrm{TeV}$ range).

The estimated uncertainties on the simulations, especially at lower energies, were historically much smaller than the uncertainties on the direct experimental measurements of the flux. However, as the current generation of neutrino detection experiments improve statistics and reduce systematic uncertainties, measuring the atmospheric neutrino flux by direct data measurements becomes increasingly useful - allowing more accurate cross-checks and feedback to the future development of the simulations, and discovery of several new effects that have been predicted by the simulations but not yet observed, such as directional asymmetries and modulation over time. Specifically this thesis presents three direct measurements of the atmospheric neutrino flux at Super-Kamiokande (SK): the directionally-integrated energy spectrum of the flux (with high statistics in the $100 \mathrm{MeV}$ to $10 \mathrm{TeV}$ range), the azimuthal spectrum, and the modulation of the flux with time over the elevenyear solar cycle. 


\section{Measurement and Results}

Measurements of the atmospheric neutrino flux were performed using the water-Cherenkov detector Super-Kamiokande [25], which has the world's largest dataset of atmospheric neutrino interactions, with an exposure of approximately $315 \mathrm{kton}$ yr. The atmospheric $v_{e}+\bar{v}_{e}$ and $v_{\mu}+\bar{v}_{\mu}$ fluxes were measured and analysed as a function of energy, azimuthal direction, and time, with a detailed analysis of systematic errors.

The energy spectra were measured in the range $100 \mathrm{MeV}$ up to $10 \mathrm{TeV}$, as shown in Fig. 1. Our measured data provided significantly improved precision up to $100 \mathrm{GeV}$, and the first data below $320 \mathrm{MeV}$. We compared our results against the predictions from several atmospheric neutrino flux models, as shown graphically in Fig. 2. A numerical comparison was performed by calculating the $\chi^{2}$ statistic, taking into account the error correlation matrix. The combined $\chi^{2}$ of the $v_{e}$ and $v_{\mu}$ fluxes (corresponding to 23 bins) was found to be 21.8, 25.6, and 30.7 for the HKKM11, Fluka, and Bartol models respectively. We see that while none of the current generation flux models are strongly inconsistent with our data, there was some preference for the HKKM11 model as the best-fit to our data. Energy spectra measurements were also obtained using separate neutrino and antineutrino enriched samples, which also showed agreement with the current flux models.

The azimuthal spectra showed an east-to-west dipole asymmetry in the neutrino flux, caused by the geomagnetic field, for both neutrino flavours at $>5 \sigma$. The plots used to determine this significance are shown in Fig. 3, from which we define the parameter $A$ as

$$
A=\frac{n_{\text {east }}-n_{\text {west }}}{n_{\text {east }}+n_{\text {west }}}
$$

where $n_{\text {east }}\left(n_{\text {west }}\right)$ represents the number of east-going (west-going) single-ring events in the SK dataset $^{1}$. This parameter is simply a measure of the dipole asymmetry strength; the significance is defined as $\Delta A / A$. Our measurement gives the discovery of the effect for the $v_{\mu}$ flux. The strength of this dipole asymmetry was furthermore shown to depend on neutrino energy and zenith as predicted by the HKKM11 model, as shown in Fig. 4.

Furthermore, an indication of a predicted shift of the dipole asymmetry angle depending on the zenith angle was seen at the $2.2 \sigma$ level, by using the parameter $B$ in the fitting

$$
k_{1} \sin (\phi+B)+k_{2}
$$

where $k_{1}$ and $k_{2}$ are free parameters, and $\phi$ is the azimuthal angle. This parameter as a function of zenith angle is shown in Fig. 5, and the significance was taken as $\sqrt{\chi_{\mathrm{flat}}^{2}-\chi_{\mathrm{MC}}^{2}}$, where $\chi_{\mathrm{MC}}^{2}$ is the $\chi^{2}$ statistic with respect to the HKKM11 MC prediction and $\chi_{\text {flat }}^{2}$ is with respect to a flat distribution (i.e. assuming no zenith dependence of the dipole angle), considering $e$-like and $\mu$-like events simultaneously. This is the first attempt at a measurement that explores the geomagnetic field effects on the neutrino flux beyond a simple east-west asymmetry. Altogether, these azimuthal measurements give confidence that the flux simulations correctly model the complicated effects of the geomagnetic field.

\footnotetext{
${ }^{1}$ Which is a sample whereby a single electron-like or muon-like track is reconstructed inside the detector, and is a very high purity $v_{e}$ or $v_{\mu}$ induced sample.
} 


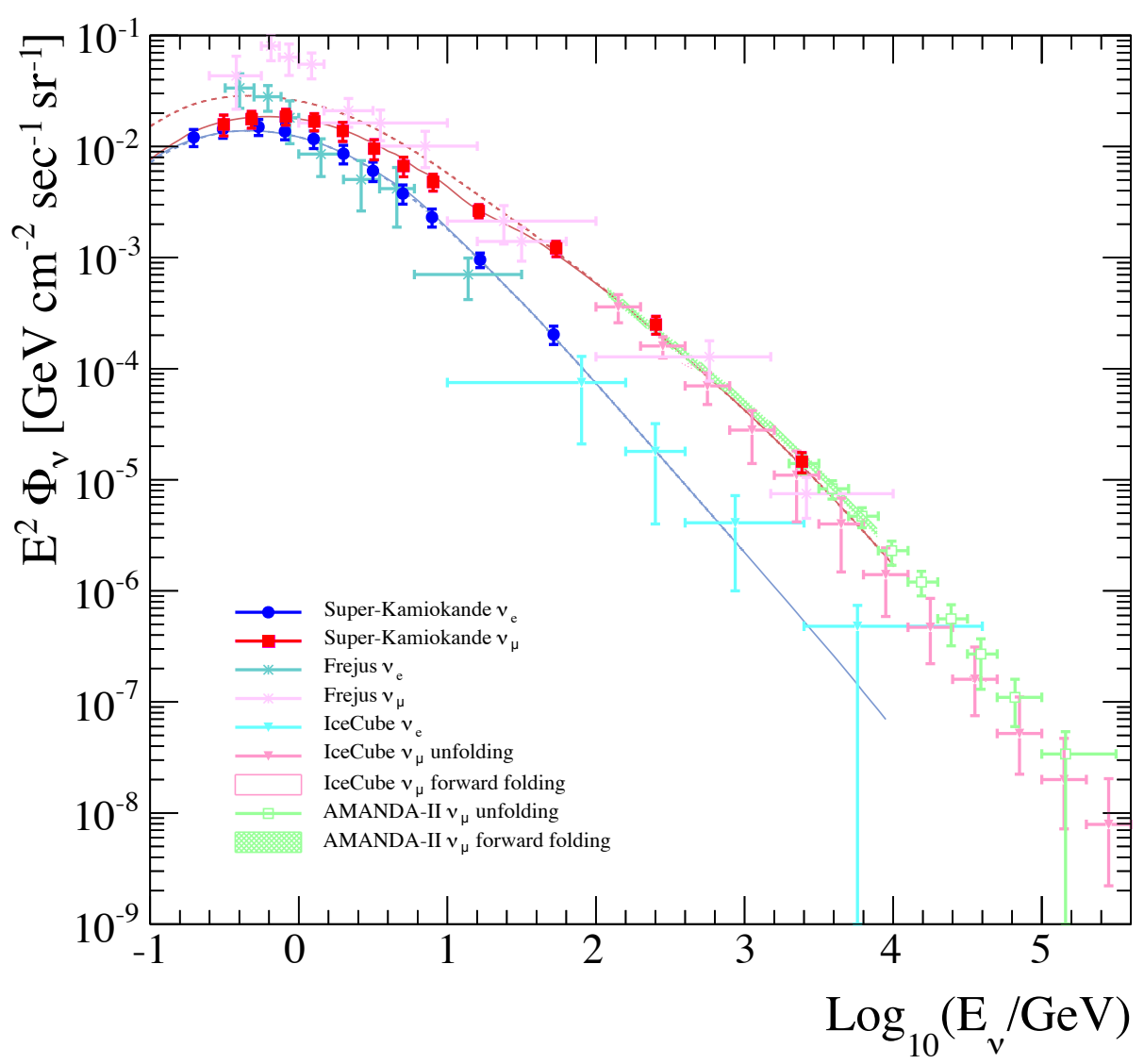

Figure 1: The measured energy spectra of the atmospheric $v_{e}+\bar{v}_{e}$ and $v_{\mu}+\bar{v}_{\mu}$ fluxes at SK, shown in comparison to measurements by Frejus [19], AMANDA-II [20, 21], and IceCube [22, 24, 23]. The HKKM11 [16] model predictions are also shown in solid (with oscillation) and dashed (without oscillation) lines.

A study of the time correlation between the atmospheric neutrino flux and the solar magnetic activity cycle was performed, where the solar activity was assumed to be correlated with the neutron flux at ground level. The type of correlation between the neutrino and neutron flux predicted by the HKKM group was calculated to have a relatively minor effect on most of the Super-Kamiokande data, but by searching over two solar maxima using approximately 14 years of data, a slight preference for such a correlation was seen at the $1.1 \sigma$ level. This correlation is shown in Fig. 6.

By separate examination of several short periods (not included in the long-term analysis due to lack of a theoretical prediction) corresponding to especially strong solar activity, from across the SK operational period for a total exposure of 7.1 days, an indication for some decrease in the atmospheric neutrino flux was seen at the $2.4 \sigma$ level.

\section{Summary and Future}

These direct measurements of the atmospheric neutrino flux tested the theoretical models with improved precision, and searched for several new physical effects not previously measured. While 


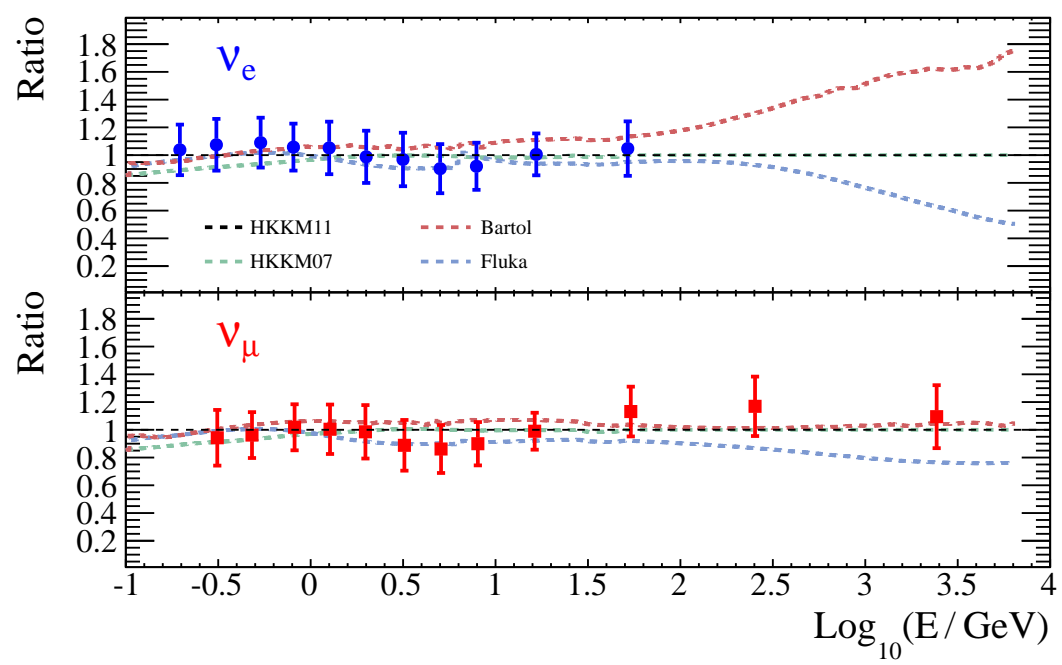

Figure 2: The measured energy spectra of the atmospheric $v_{e}+\bar{v}_{e}$ and $v_{\mu}+\bar{v}_{\mu}$ fluxes at SK, divided by the predictions from the HKKM11 flux model. The ratios of several other flux models to the HKKM11 model are also shown.
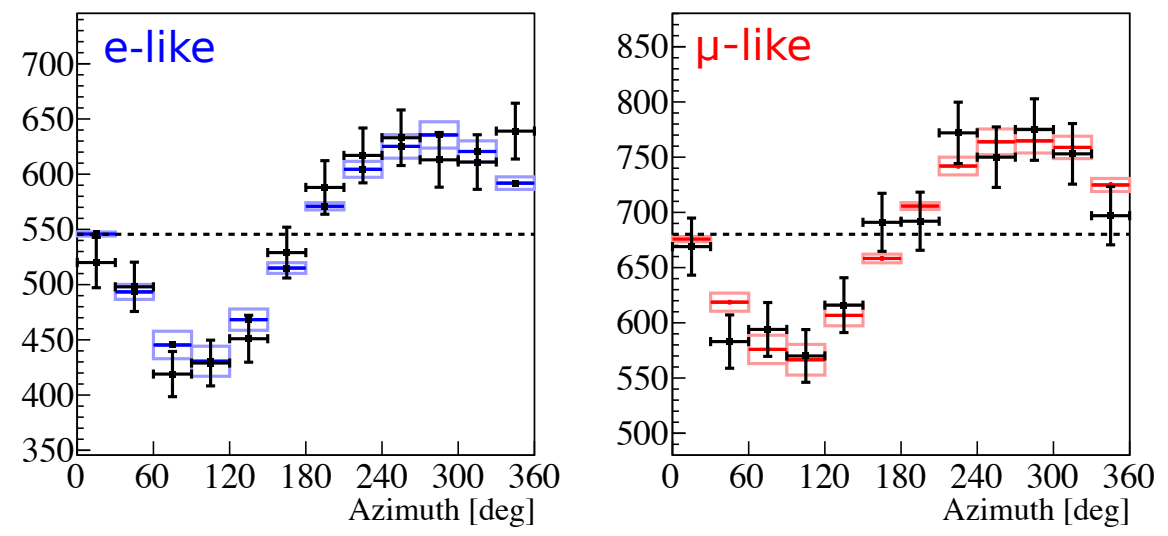

Figure 3: A subset of the single-ring $e$-like and $\mu$-like neutrino induced events in the SK-I to SK-IV data (points) and MC (boxes), optimized to show the east-west asymmetry by selecting events with reconstructed energy $0.4<E_{\text {rec }}<3.0 \mathrm{GeV}$ and incoming angle $-0.6<\cos ($ zenith $)<0.6$.

only one new measurement reached the $5 \sigma$ discovery level, even those with relatively low significance are interesting indications that suggest further study by the next generation of neutrino detectors. Our measurements are in general consistent with the current generation flux models, which gives confidence in our understanding and modeling of the atmospheric neutrino flux.

In future, combining our measurements with those from other current generation neutrino detectors, which are sensitive at distinct but overlapping energy regions, even more accurate data may be obtained. For example, constraints given by the improved precision of our energy spectra measurement combined with other measurements at higher energies can help to accurately deter- 

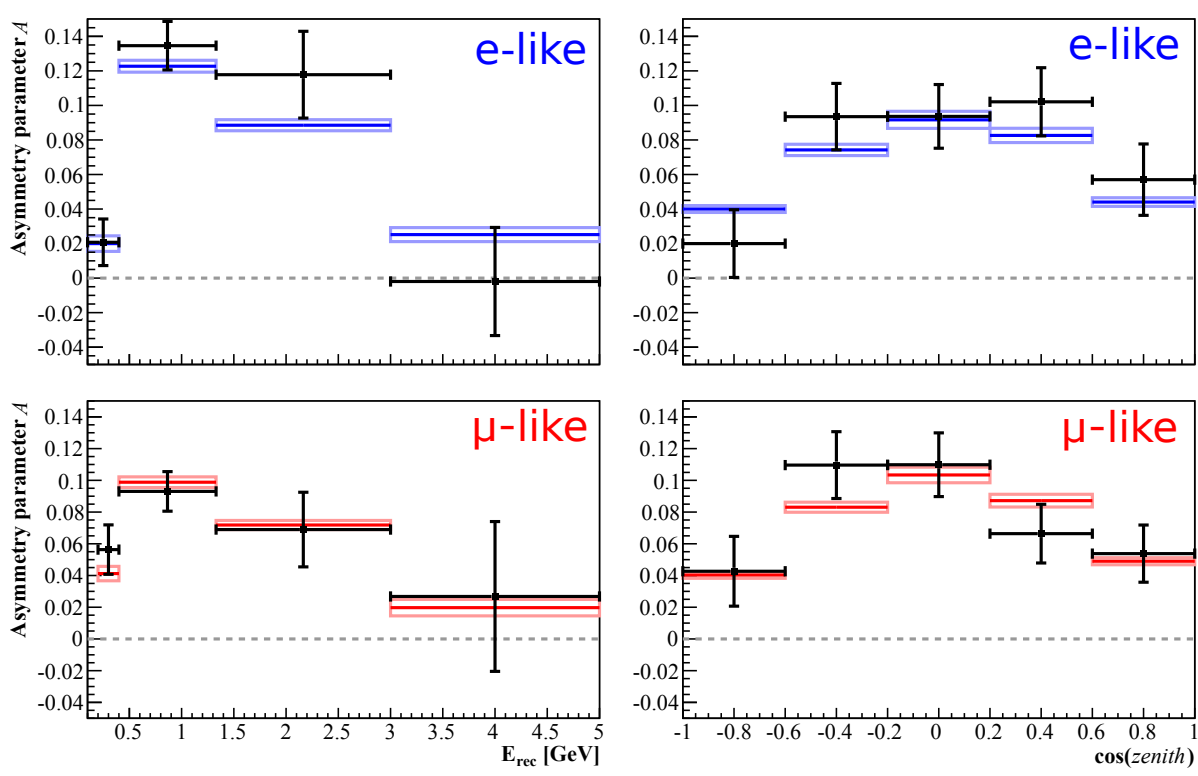

Figure 4: The $A$ parameter (described in the text) for single-ring $e$-like and $\mu$-like neutrino induced events in the SK-I to SK-IV data (points) and MC (boxes).
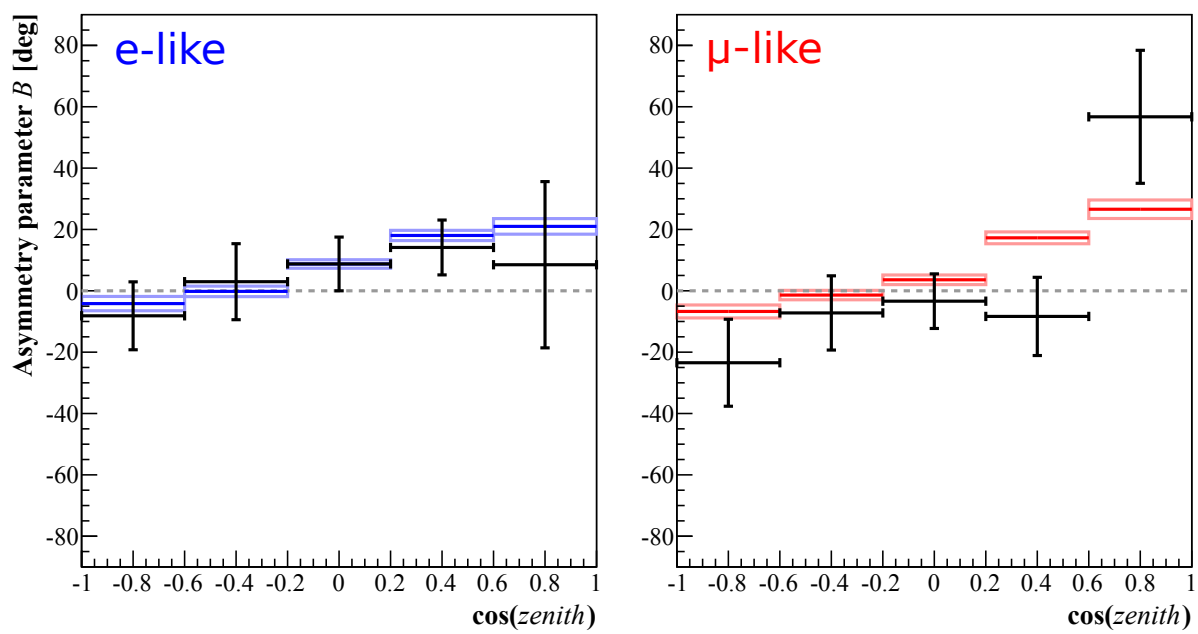

Figure 5: The $B$ parameter (described in the text) for single-ring $e$-like and $\mu$-like neutrino induced events in the SK-I to SK-IV data (points) and MC (boxes). 

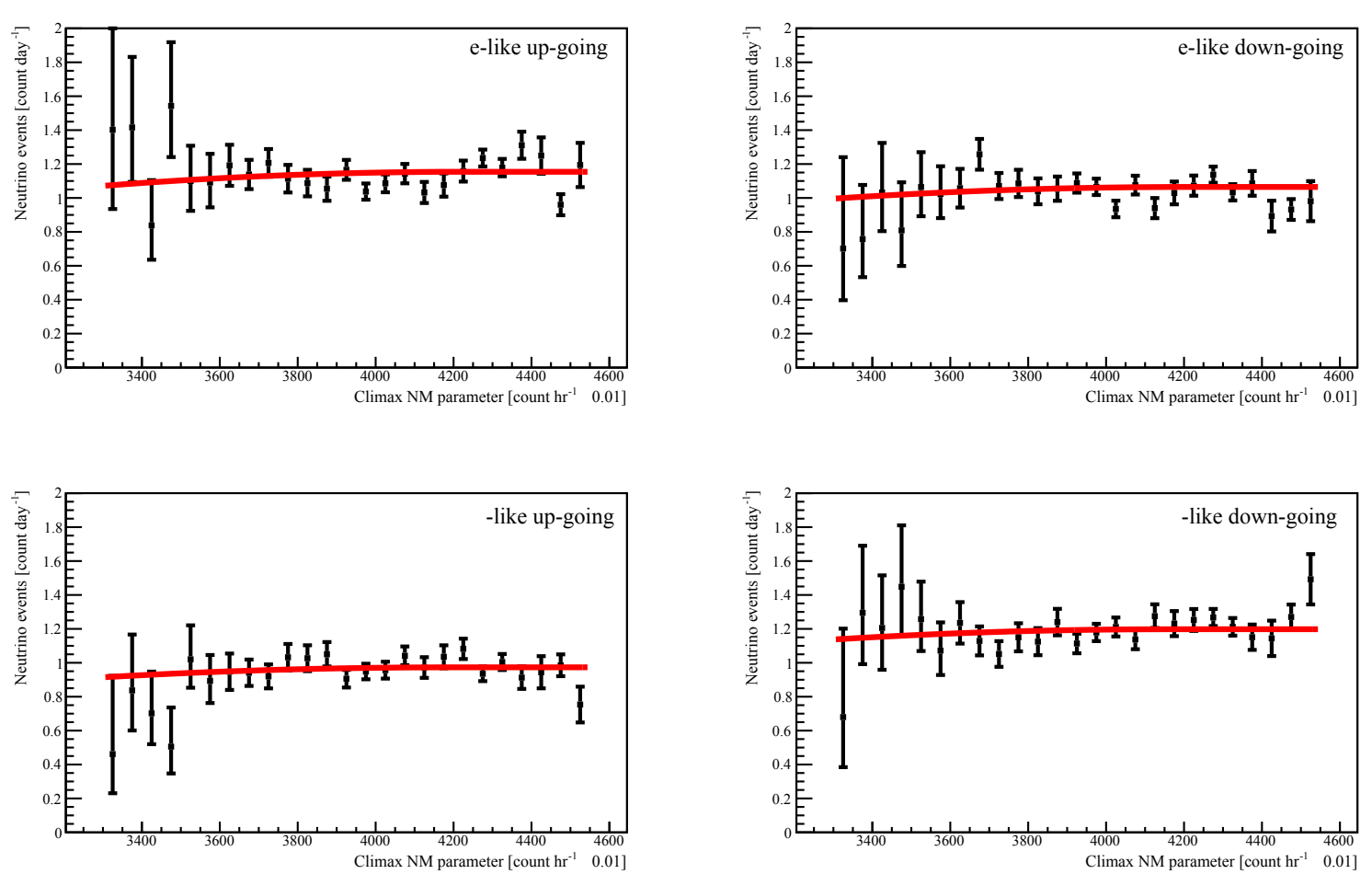

Figure 6: Events from four SK neutrino data samples, simultaneously fit against the predicted form of a solar activity correlation according to the HKKM model, where "Climax NM parameter" is a parameter based on the neutron count at various monitor stations around the Earth. Although hard to see by eye, the predicted form of the function differs depending on the data sample.

mine the astrophysical neutrino spectra. In general, further improvements to the atmospheric flux measurements could also provide feedback to the flux simulations, and further a better systematic understanding of the atmospheric neutrino flux as both a background and a signal source.

\section{References}

[1] C. V. Achar et al. Detection of muons produced by cosmic ray neutrinos deep underground. Phys. Lett. 18 (1965) 196.

[2] F. Reines, et al. Evidence for High-Energy Cosmic-Ray Neutrino Interactions. Phys. Rev. Lett. 15 (1965) 429.

[3] Y. Fukuda et al. Evidence for Oscillation of Atmospheric Neutrinos. Phys. Rev. Lett. 81 (1998) 1562.

[4] R. Wendell et al. Atmospheric neutrino oscillation analysis with sub-leading effects in SuperKamiokande I, II, and III. Phys.Rev. D81 (2010) 092004. 1002.3471.

[5] J. Hosaka et al. Solar neutrino measurements in super-Kamiokande-I. Phys.Rev. D73 (2006) 112001. hep-ex/0508053. 
[6] B. Aharmim et al. Electron energy spectra, fluxes, and day-night asymmetries of B-8 solar neutrinos from measurements with $\mathrm{NaCl}$ dissolved in the heavy-water detector at the Sudbury Neutrino Observatory. Phys.Rev. C72 (2005) 055502. nucl-ex/ 0502021.

[7] S. Abe et al. Precision Measurement of Neutrino Oscillation Parameters with KamLAND. Phys.Rev.Lett. 100 (2008) 221803. 0801.4589.

[8] F. An et al. Observation of electron-antineutrino disappearance at Daya Bay. Phys.Rev.Lett. 108 (2012) 171803. 1203.1669.

[9] J. Ahn et al. Observation of Reactor Electron Antineutrino Disappearance in the RENO Experiment. Phys.Rev.Lett. 108 (2012) 191802. 1204.0626.

[10] Y. Abe et al. Indication for the disappearance of reactor electron antineutrinos in the Double Chooz experiment. Phys.Rev.Lett. 108 (2012) 131801. 1112.6353.

[11] M. Ahn et al. Measurement of Neutrino Oscillation by the K2K Experiment. Phys.Rev. D74 (2006) 072003. hep-ex/0606032.

[12] P. Adamson et al. Measurement of the neutrino mass splitting and flavor mixing by MINOS. Phys.Rev.Lett. 106 (2011) 181801. 1103.0340.

[13] K. Abe et al. Precise Measurement of the Neutrino Mixing Parameter $\theta_{23}$ from Muon Neutrino Disappearance in an Off-Axis Beam. Phys.Rev.Lett. 112 (2014) (18) 181801. 1403.1532.

[14] K. Abe et al. Observation of Electron Neutrino Appearance in a Muon Neutrino Beam. Phys.Rev.Lett. 112 (2014) 061802. 1311.4750.

[15] K. Abe et al. Physics Potential of a Long Baseline Neutrino Oscillation Experiment Using J-PARC Neutrino Beam and Hyper-Kamiokande. PTEP (2015). 1502.05199.

[16] M. Honda, et al. Improvement of low energy atmospheric neutrino flux calculation using the JAM nuclear interaction model. Phys. Rev. D83 (2011) 123001.

[17] G. Battistoni, et al. The FLUKA atmospheric neutrino flux calculation. Astroparticle Physics 19 (2003) (2) 269 . ISSN 0927-6505.

[18] G. Barr, et al. Three-dimensional calculation of atmospheric neutrinos. Phys. Rev. D70 (2004) 023006.

[19] K. Daum. Determination of the atmospheric neutrino spectra with the Frejus detector. $Z$. Phys. C66 (1995) 417.

[20] R. Abbasi et al. Determination of the Atmospheric Neutrino Flux and Searches for New Physics with AMANDA-II. Phys.Rev. D79 (2009) 102005. 0902.0675.

[21] R. Abbasi et al. The Energy Spectrum of Atmospheric Neutrinos between 2 and $200 \mathrm{TeV}$ with the AMANDA-II Detector. Astropart.Phys. 34 (2010) 48. 1004.2357.

[22] R. Abbasi et al. Measurement of the atmospheric neutrino energy spectrum from $100 \mathrm{GeV}$ to $400 \mathrm{TeV}$ with IceCube. Phys. Rev. D83 (2011) 012001. 1010 . 3980.

[23] M. Aartsen et al. Measurement of the Atmospheric ve Flux in IceCube. Phys. Rev. Lett. 110 (2013) 151105.

[24] R. Abbasi et al. A Search for a Diffuse Flux of Astrophysical Muon Neutrinos with the IceCube 40-String Detector. Phys.Rev. D84 (2011) 082001. 1104.5187.

[25] Y. Fukuda et al. The Super-Kamiokande detector. Nucl.Instrum.Meth. A501 (2003) 418. 